\title{
TIME-RESOLVED INVESTIGATION OF THE COMPENSATION PROCESSS OF PULSED ION BEAMS
}

\author{
A. Jakob, H. Klein, A. Lakatos, O. Meusel, J. Pozimski
}

\section{Abstract}

A LEBT system consisting of an ion source, two solenoids, and a diagnostic section has been set up to investigate the space charge compensation process due to residual gas ionization [1] and to study experimentally the rise of compensation. To gain the radial beam potential distribution time resolved measurements of the residual gas ion energy distribution were carried out using a Hughes Rojanski analyzer [2,3]. To measure the radial density profile of the ion beam a CCD-camera performed time resolved measurements, which allow an estimation the rise time of compensation. Further the dynamic effect of the space charge compensation on the beam transport was shown. A numerical simulation under assumption of selfconsistent states [4] of the beam plasma has been used to determine plasma parameters such as the radial density profile and the temperature of the electrons. The acquired data show that the theoretical estimated rise time of space charge compensation neglecting electron losses is shorter than the build up time determined experimentally. An interpretation of the achieved results is given.

\section{INTRODUCTION}

Magnetic low energy beam transport (LEBT) systems enabel space charge compensated transport of high perveance ion beams in comparison to electrostatic focussing systems, which suffer from the high beam energy and the influence of the space charge forces on the beam. The magnetic LEBT suffers from the rise time of space charge compensation of pulsed ion beams.

Variing space charge forces of a pulsed ion beams lead to a shift of the focus and therefore to a mismatch of the following accelerator section. To investigate the effects of the rise time of compensation on the transport of ion beams a LEBT section with non-destructive diagnostics was set up in Frankfurt [5].

\section{EXPERIMENTAL SETUP}

Fig 1 shows the experimental set up at the Institut fuer Angewandte Physik (IAP) to investigate the behaviour of an ion beam (i.g. $10 \mathrm{keV}$ ) transported through a LEBT section. The LEBT has a length of approximately three meters and consist of an ion source, a first small diagnostic section, to measure the beam current directly behind the extraction system, two solenoids $\left(\mathrm{B}_{\max }=0.8\right.$ $\mathrm{T})$ and a following diagnostic section with different diagnostic tools, to investigate the development of the compensation process. In the first step of the investigation of the compensation process the ion sorce was run in DC mode and the ion beam was decompensated in pulsed mode by a decompensation electrode electrode, which was biased at $350 \mathrm{~V}$, with a repetition rate of $1 \mathrm{kHz}$, a decompensation time of $150 \mu \mathrm{s}$ (decompensation biased at $350 \mathrm{~V}$ ) and a compensation time of $850 \mu$ s (grounded electrode).

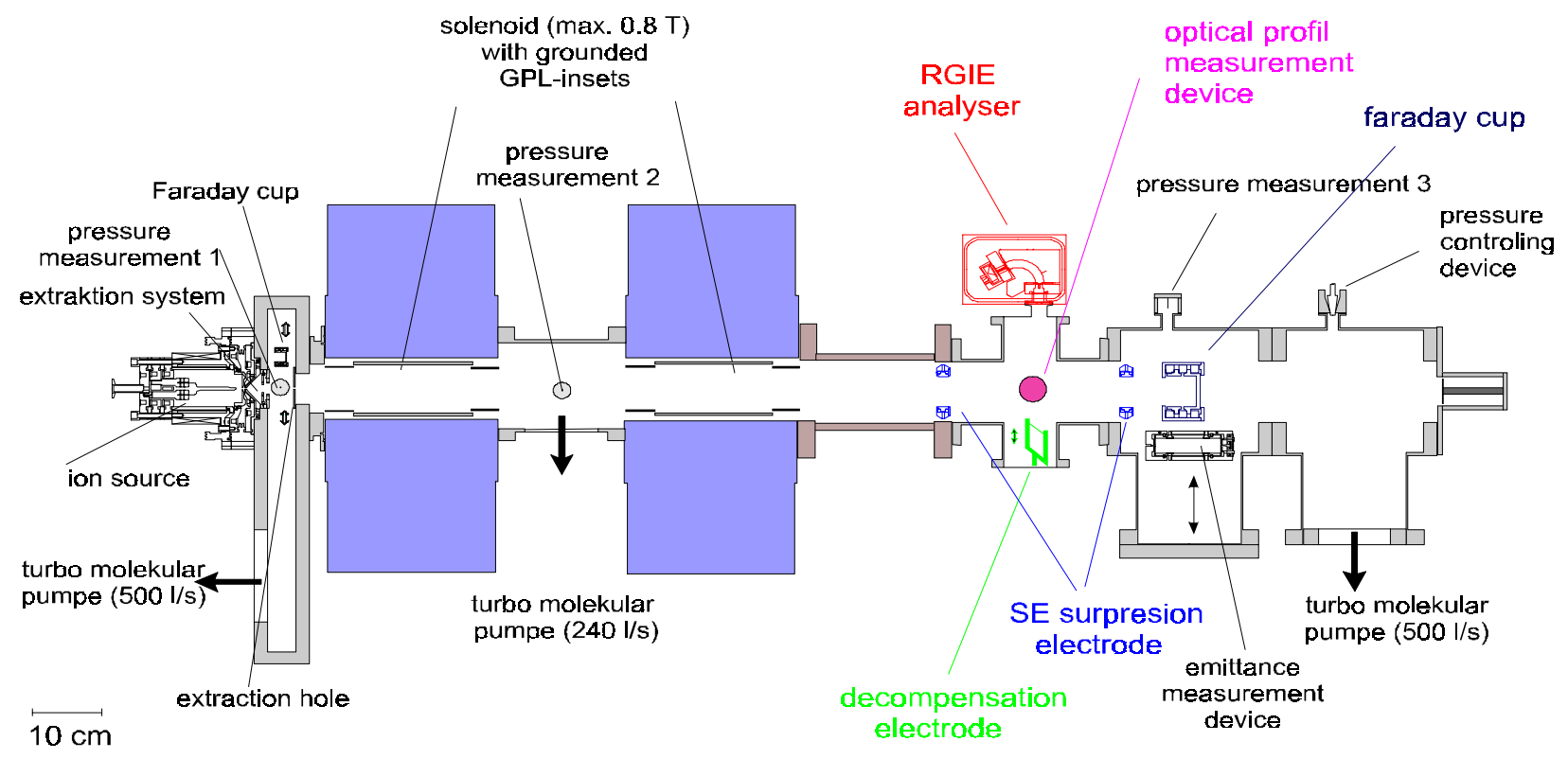

Fig1. : Frankfurt Low Energy Beam Transport (LEBT) section 
As decompensation electrode a moveble wire was used. Thus it was possible to move the electrode to an definite place, which can be observe by the CCD camera, therefore we can be sure that the beam does not touch the wire, which has a strong effect on the compensation process by the production of secundary electrons. In the second step the ion source was run in pulsed mode, with a repetition rate of $1 \mathrm{kHz}$ and a duty cycle of $90 \%$. To observe the rise time of the ion source, the current measuremets by the second Faraday cup in the LEBT (fig.1) were done using an oscilloscope. Fig. 2 shows the result of such a measurement.

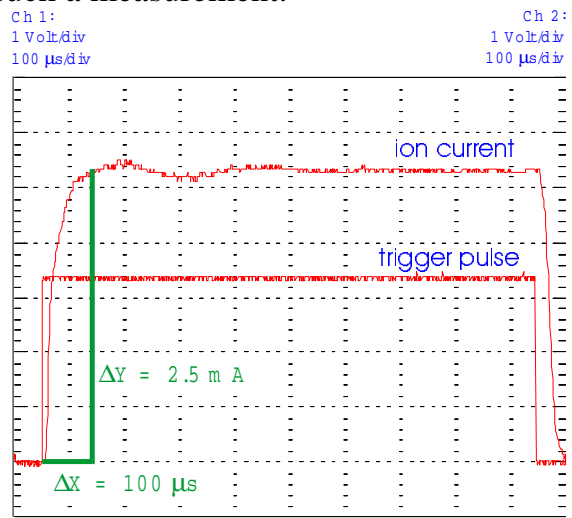

Figure 2: Beam current measurement of a pulsed ion beam

For a good comparability of the measurements all used diagnostics were placed at the same area, the CCD camera and the time-resolving residual gas ion energy analyser (RGIEA) were arranged orthogonally and the Faraday cup a few $\mathrm{cm}$ behind. The decompensation electrode also was placed orthogonally to the diagnostic devices to reach a local decompensation right at the region of interrest. To find a better understanding of the data measured at the pulsed ion beam, the measurements at a pulsed decompensated DC ion beam were used as reference.

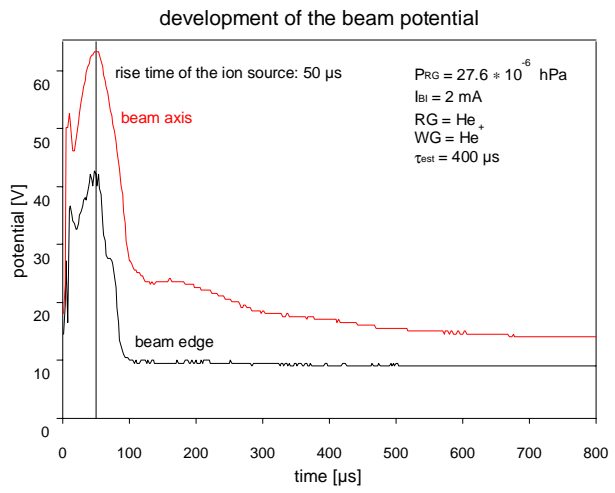

Figure 3: Temporal development of potentials of beam axis and beam edge.

The residual gas ions (RGI) produced by the interaction of beam ions and residual gas are expelled radially by the self field of the beam. Under assumption of neglectable start energy, the kinetic energy of the RGI corresponds to the beam potential at the point of production. The energy distribution of the residual gas ions contains information on the radial beam potential distribution and thereby on the degree of compensation. For the time-resolving investigation of the build-up time of space charge compensation a RGIEA with an inserted single particle detector (channeltron) [5] was used. The time resolution was limited by the data acquisition to 2 $\mu \mathrm{s}$.

\section{MEASUREMENTS}

Fig.3 shows the development of the beam potentials using pulsed beam operation obtained by the intersection point of the dynamic energy spectra and the $5 \%$ baseline (5\% threshold) corresponding to the potential at the beam axis and the beam edge.

The CCD camera was used to observe the light emitted by the residual gas excited by the interaction of the beam ions with the residual gas molecules. Therefore one get the time development of the beam ion density under the assumption that the intensity of the emitted light is strongly correlated to the beam ion density. From the Abel transformation of the data gained from CCD measurements one can obtain the denstity profile of the ion beam. Fig 5 shows the development of the radius of the ion beam estimated from the $20 \%$ threshold of the measured beam ion density profiles.

Fig.2 shows, that the ion source needs approximately $100 \mu$ s to reach a state of constant ion generation, then a current of $2 \mathrm{~mA}$ was measured. At $50 \mu$ s nearly $80 \%$ of the maximum current is reached, after $50 \mu$ s the current increases substantially slower. This can also be read off from fig.3. The potential increases up to $50 \mu \mathrm{s}$, then it decreases due to the compensation of the space charge forces of the ion beam.

In the first $50 \mu$ s the rise of the ion beam density dominats the development of the potential of the beam, due to the increasing ion production rate. Afterwards the compensation process dominates the potential development, which yields to a continous potential depression. Fig.4 shows the development of the beam potential for a pulsed decompensated $\mathrm{He}^{+}(10 \mathrm{kV}, 2 \mathrm{~mA})$ DC ion beam under same conditions (residual gas pressure, beam current, duty cycle).

In comparison to the potential development of a pulsed ion beam, the potential depression starts just at the time when the compensation process begins. The maximum potential is by a faktor 1.5 higher in comparison to the pulsed operation mode. Both measurements (fig.2 and fig.4) show that after approximately $500 \mu \mathrm{s}$ the process of potential depression is completed. Fig.5 also shows that after $500 \mu$ s the development of the beam radius, 
obtained from the CCD profile measurements, is completed, no further shrinking can be ascertain.

The comparison of both measurements shows, that in case of a pulsed ion beam the compensation process runs faster than in the case of a pulsed decompensated DC beam.

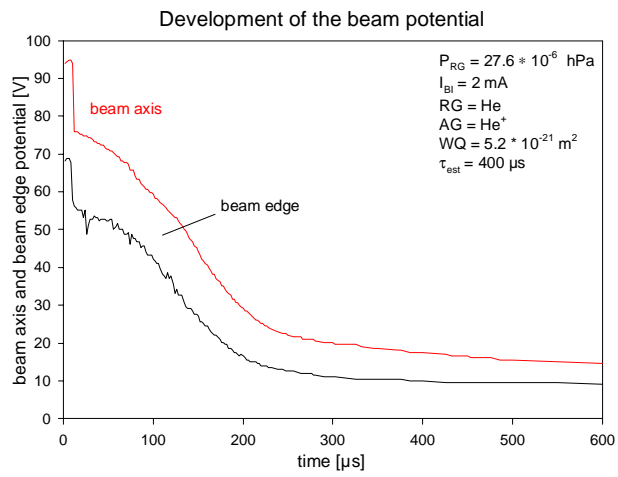

Figure 4: Temporal development of beam potential In case of DC operation mode the potential drop inside the ion beam at the begining of the compensation process has a substantially higher value in comparison to the pulsed operation mode of the ion source. Due to this effect two processes accur.

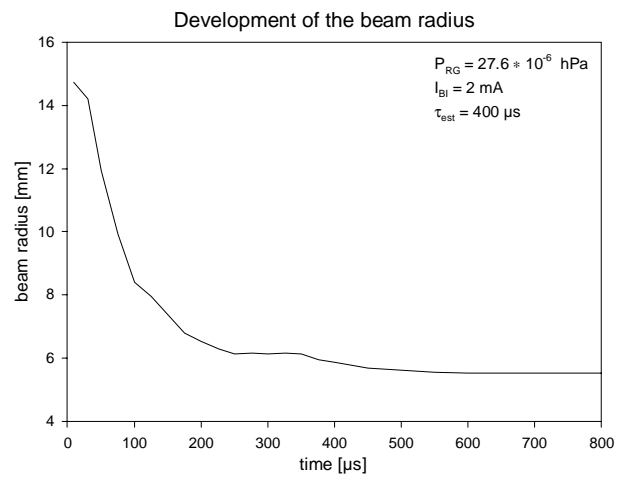

Figure 5: Temporal development of beam radius of a DC ion beam, which was decompensated in pulsed mode.

The compensation electrons produced at the beginning of the compensation process are trapped in the potential well. Due to the deeper potential drop in case of DC operation mode electrons of higher energy can be trapped. In case of pulsed mode faster electrons instantly get lost. The fast electrons increase the temperature of the trapped electrons. Due to the higher temperatur it becomes harder for the decreasing beam potential to trap the electrons, which yields to a prolongation of the compensation process. Secondly the trapped electrons are accelarated in the potential gradient and therefore gain more kinetic energy in case of DC operation mode then in comparison to the pulsed operation mode, which yields to a higher averrage energy of the trapped electron ensemble and therefore again to a prolongation of the compensation process. In the present status of the analysis we do not know which process dominates. This needs further studies and a precise investigation of the results of the self consistent simulations.

Fig 6 shows the development of the radius of a pulsed ion beam. It shows the same behaviour like in fig.5. (The fluctuation of the beam radius belongs to fluctuation of the measured data in combination with the data evaluation.)

The present considerations shows, that the process of space charge compensation of a pulsed as well as of a periodically decompensated $\mathrm{DC} \mathrm{He}^{+}(10 \mathrm{keV}, 2 \mathrm{~mA})$ ion beam is finished after approximatly $500 \mu \mathrm{s}$. The rise time of compensation can be estimated [1] to $400 \mu$ s assuming constant electron production rate and neglecting electron losses.

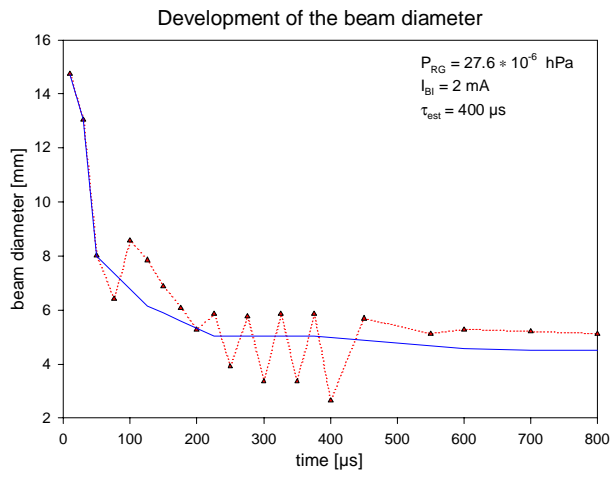

Figure 6: Temporal development of the beam radius of a pulsed ion beam, obtained from measured data (dotted line) and smoothed curve (full line).

\section{FUTURE WORK}

To get a precise estimation of the rise time of compensation, we have to await the results of the data avaluation using self consistent numerical simulation, which is in process. Furtheron the time-resolved measurements will be performed on a pulsed ion beam of other ion species and ions of higher energy.

The time development of the kinetic and potential energy of the compensation electrons will be analyzed using the numerically results of the simulation. The achieved particle and energy balance leads to a better understanding of particle loss processes, which is important for the investigation of the compensation processses.

\section{REFERENCES}

[1] A.J.T. Holmes, Theoretical and experimental study of space charge intense ion beams., Phys. Rev. A 19 (1979), p 389

[2] A.L. Hughes, V. Rojanski, On the analysis of electronic velocities by electrostatic field, Phys. Rev. 34 (1929) 291

[3] R. Dölling, Raumladungskompensation driftender intensiver Strahlen niederenergetischer Ionen und Techniken zu ihrer Vermessung, Dissertation, IAP, JWG-University, Frankfurt, 1994

[4] J. Pozimski et.al., „Determinarion of Electron Temperature in Partial Space Charge Compensated High Perveance Ion Beams“, Proc. International Symposium on Heavy Ion Inertial Fusion, Il Nuovo Cimento 106A N.11, Frascati, Italy, May 1993, pp.1713

[5] A.Jakob, Study of compensation process of ion beams, Proc XIX LINAC Conf. Chicago 1998 